\title{
Sound Propagation in Curvilinear Space Time
}

The theories of acoustics and the derivation of the equations of acoustics such as the. Helmholtz wave equation, KZK equation, Burgers equation, Westervelt equation, Christoffel equation for crystal acoustics are all based on the flat space time or Minkowski space time. This limits sound propagation only to $2 \mathrm{D}$ plane instead of $3 \mathrm{D}$ space. Also in so doing the equations will come out with the special theory of relativity. In the extension of the calculations to the curvilinear space time, general relativity will be involved. This will also include the gravitational force. Also energy and momentum will affect the curvature of the curvilinear space time.

The scenario of the acoustic field equation without involving the gravitational force is like the situation in a space station. Sofar the acoustical cloaking is the first example of sound propagation in a curvilinear space time. Curvilinear space time will be a new platform for the derivation of the acoustic equations. There are numerous calculations in acoustics based on curvilinear coordinates. But these are only for the description of the geometrical structure of the objects under consideration. Also in acoustical cloaking only one aspect of the general theory of relativity is concerned. That is only the curvilinear coordinates aspect of the problem is considered. The property of gravitational force is not considered. It is of interest to mention that in his original paper on the foundation of general relativity, Einstein mentioned curvilinear space time on two areas of physics, that is electrodynamics and hydrodynamics which are related to acoustics.

Besides acoustical cloaking, an area which is concerned with curvilinear space time is acoustic radiation force (ARF). ARF has wide applications to drug delivery, microfluidics, acoustical imaging, force of levitation etc. Normally the gravitational force is neglected. The usual acoustics field equation is derived from Newton's equation of motion which is based on flat space time. The proper procedure is to use the Einstein field equations and action principles to derive the ARF. This will allow the interaction of the ARF with the gravitational force.

Another area to be considered is acoustical imaging. For the case of curvilinear space time, the gravitational force term has to be added to the source term on the right hand side of the inhomogeneous Helmholtz equation. Also the multiple scattering in an inhomogeneous medium now takes place in a curvilinear space time. A form of acoustical imaging known as elasticity imaging will be affected by the inclusion of the gravitational force as the stress tensor. This will become part of the energy momentum tensor in the Einstein field equations. ii

In the field of nonlinear acoustics in curvilinear space time, all the field equations like KZK equation, Burgers equation, Westervelt equation will have to be derived using the Einstein field equations relating the energy momentum tensor and the curvature tensor. As a consequence, the gravitational force will become an additional term in the equations along with terms relating to diffraction, refraction, and nonlinearity. Curvilinear space time is the umbrella of nonlinearity.

Elasticity in the curvilinear space time is an interesting area as the stress tensor is part of the energy momentum tensor of the Einstein field equations. The gravitational force here will in the term involving the body force. Here the stress and strain will all be expressed in tensor forms for substitution in the Einstein field equations.

In piezoelectricity, for curvilinear space time, gravitational force will become an additional force involved in the interaction between electromagnetic forces and acoustic forces. Now there will be three forces involved in the interaction.

In the electron-phonon interaction and phonon-phonon interaction in sound propagation in solids, there will be an additional gravitational force interaction in the curvilinear space time platform. This will be a form of the unification of quantum field theory and general relativity which points towards the theory of everything.

The above subfields of acoustics have to be considered when one rewrites acoustics equations using the curvilinear space time platform. In view that Einstein purposely singled out hydrodynamics which is related to acoustics as an area in physics for the extension of fundamental physics theories to the curvilinear space time, it is now time to work on acoustics based on this theoretical platform. It is to be noted that for all applications the equations have to be derived again using the Einstein field equations. If only a force of gravity term is added, this will amount to just a Newtonian treatment or flat space time.

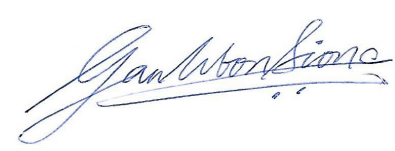

Woon Siong Gan 